\title{
Product Conceptual Design Based On Tacit Knowledge and Ontology
}

\author{
Zhang Jianmin ZHOU Jun \\ (College of Mechanical Engineering, Shanghai University of Engineering Science, Shanghai)
}

\begin{abstract}
With the coming of knowledge economy era, The traditional product design knowledge can not fully meet the user's demands for products. Improving the design quality of the product is the primary way to maximize the benefits, and the product conceptual design directly determines the quality of product design, The users'tacit knowledge is the source of the product conceptual design, therefore, the application of product conceptual design based on the Users' Tacit Knowledge was come up. On the premise of understanding the concept of the users'tacit knowledge, we classify the product requirement From the perspective of the users'demands, And using the method of thinking aloud and the method of communication to acquire tacit knowledge which is hidden in the users' mind, knowledge modeling of product conceptual design is based on the Ontology, and making the tacit knowledge which we acquired structural and explicit, and directly making the knowledge used in product conceptual design. Finally, this method is applied to product conceptual design of the multifunctional cleaning machine of small advertising paper, this method is proved feasible.
\end{abstract}

Keywords: product conceptual design; the users' tacit Knowledge; ontology; Knowledge modeling; tacit knowledge explication

\section{Introduction}

Tacit knowledge is a concept proposed by Michael (Michael Polanyi) in the field of Philosophy in 1958 ${ }^{[1,2]}$. He believes that the tacit knowledge is the carrier, exist in the brain, in a particular situation, it is difficult to use the language to express the knowledge, is an important part of knowledge innovation, mainly from the emotional, cognitive or experience etc.. The application of tacit knowledge has been involved in management, medicine, education and other aspects; and with the continuous development of modern industrial technology, the current domestic and foreign experts and scholars have begun to focus on the tacit knowledge of the designer or user, and it is widely applied in product design, such as: Japan knowledge management expert Nonaka Fujiro (Ikujiro Nonaka SECI) proposed process of explicit knowledge and tacit knowledge conversion, knowledge to solve the problem of the transformation of tacit explicit; OSGOOD proposed the semantic differential method, through the learning object (including the product color, shape) semantics, will psychological reactions of consumers in the Likert scale, and then use the related mathematical methods data processing and analysis, provide a reliable method for product conceptual design ${ }^{[3]}$; Singapore ZHAI and so on, based on the advantage of rough set method to improve user satisfaction for product design, and completed the mobile phone design ${ }^{[4]}$; Luo Wei, Pan Yunhe, Zhu Shang Shang, put forward the idea of tacit knowledge in product design, which provides an important method for the tacit knowledge ${ }^{[5]}$; Liu Zheng, Lu Na, Sun Lingyun et al. Propose a tacit knowledge acquisition method for conceptual design process ${ }^{[6]}$; Chen Youling and Shaw put forward the tacit knowledge acquisition in product design, and put forward the concept of "knowledge cell" ${ }^{[7]}$. At present, the research of tacit knowledge is the main method to get the domestic and foreign scholars in tacit knowledge and tacit knowledge is applied to the design of products, and for the explicitness of tacit knowledge, is still not mature, most of the methods are in the expression of knowledge in fuzzy state under the lead after dominance the application of the knowledge of the value is not high, often cannot be directly applied to the product conceptual design.

So, this paper proposes customer tacit knowledge in the application of product concept design, through access to the user for the perceptual evaluation of product adjectives, and based on user implicit knowledge storage mode, users' tacit knowledge and will get into products abstract feature elements, and then enter the product concept ontology model is transformed into a system of clear, the knowledge, which directly applied to the product conceptual design. At the same time, this study can also be used as the reference of knowledge management and product design.

\subsection{Tacit knowledge in product conceptual design}

II. Product conceptual design based on user's tacit knowledge

Product conceptual design is according to the needs of users, the product function analysis, and the total function further divided each sub carrier to realize the structure function of the general function and the function, and also include the function of technology principle scheme to realize the function of sub. Therefore, the essence of product conceptual design is the mapping from function to structure, which includes functional knowledge, 
structural knowledge, and the knowledge of how to realize the function.

Product design is a service for the user, the user needs the product, and the evaluation is very important, but also the tacit knowledge is the designer at the beginning of the beginning of the product design must understand, also is the user of tacit knowledge. The user in understanding the products before, will the past usage and knowledge based on experience, often produce a "impression" in the brain, such as "what it should be" function ", it looks like what" such as $4 \mathrm{~W} 1 \mathrm{H}$ (what, when, who, where, how) of the problem; when to accept products information, users usually use some adjectives, such as "ordinary" and "fine" and "light" and "simple" to describe ${ }^{[8]}$. The perceptual evaluation of these adjectives is also the tacit knowledge of the user.

\subsection{The strategy of acquiring tacit knowledge}

Strategy 1: according to the definition of tacit knowledge, we can know that ${ }^{[9]}$ : the acquisition of tacit knowledge is the process of transferring the knowledge which is difficult to express objectively from one person to another. The main body of knowledge here refers to the user or in the field of product design, has a unique insight, rich experience of the designer; similar to know, knowledge object is also so. From Figure 1 knowledge conversion chart you can see, when the object of knowledge accumulation related knowledge, will have their own experiences and views, at this time, and can be sent to the body of knowledge, a long time, each other is relationship with accepted, only when knowledge object in the physical body of knowledge based entirely on, tacit knowledge can be transformed into explicit knowledge.

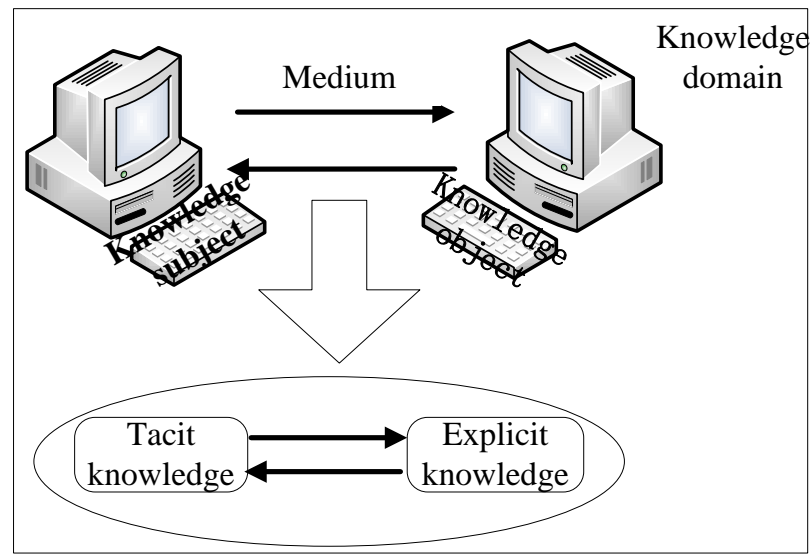

Fig.1 Knowledge conversion diagram

Strategy 2: Based on the strategy of the 1, it is known that in order to obtain the tacit knowledge, the tacit knowledge of the subject must be transferred to the object of knowledge. So this paper will use the questionnaire sampling experiment, which allows users according to their own understanding of the product to choose perceptual adjectives (adjectives of the selected may consult experts or designer), then the designer analyze and extract the product characteristics of abstract elements, as shown in figure 2 :

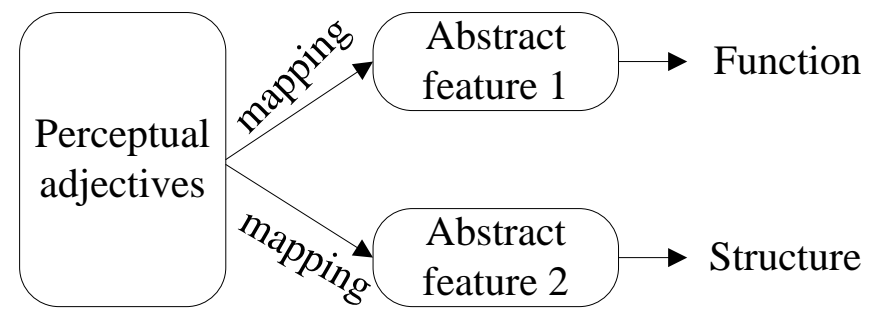

Fig.2 Extraction of the abstract feature elements from the user's perceptual adjectives

\subsection{The explication of tacit knowledge}

Ontology originated in the field of philosophy, is an entity that is application analysis and modeling method of the Hugh theory to a field, a field that is in the real world are abstracted as relationships between a set of concepts ${ }^{[10]}$.

At the same time, as a kind of content oriented concept system tool ontology can express the relation between conceptual entities in a very clear way, the knowledge expression of more systematic and structured ${ }^{[11]}$. While the tacit knowledge makes the previous methods to express the knowledge most not systematic, and the knowledge expression in the fuzzy state, often can not fully knowledge clearly, lead to waste phenomenon of 
knowledge and using knowledge is not clear phenomenon is quite serious, so this paper will be obtained by extracting tacit knowledge abstract the product features, and then enter the product concept ontology model of knowledge explicit, systematic, and clarity of expression, which can be directly applied in product conceptual design. In order to improve the efficiency of the use of knowledge, reduce the workload of designers, to avoid the waste of knowledge. The product concept ontology model is shown in Figure 3 below: among them, inheritance relationship: Is-a, part and whole relation: Part-0f, attribute relation: Attribute-of.

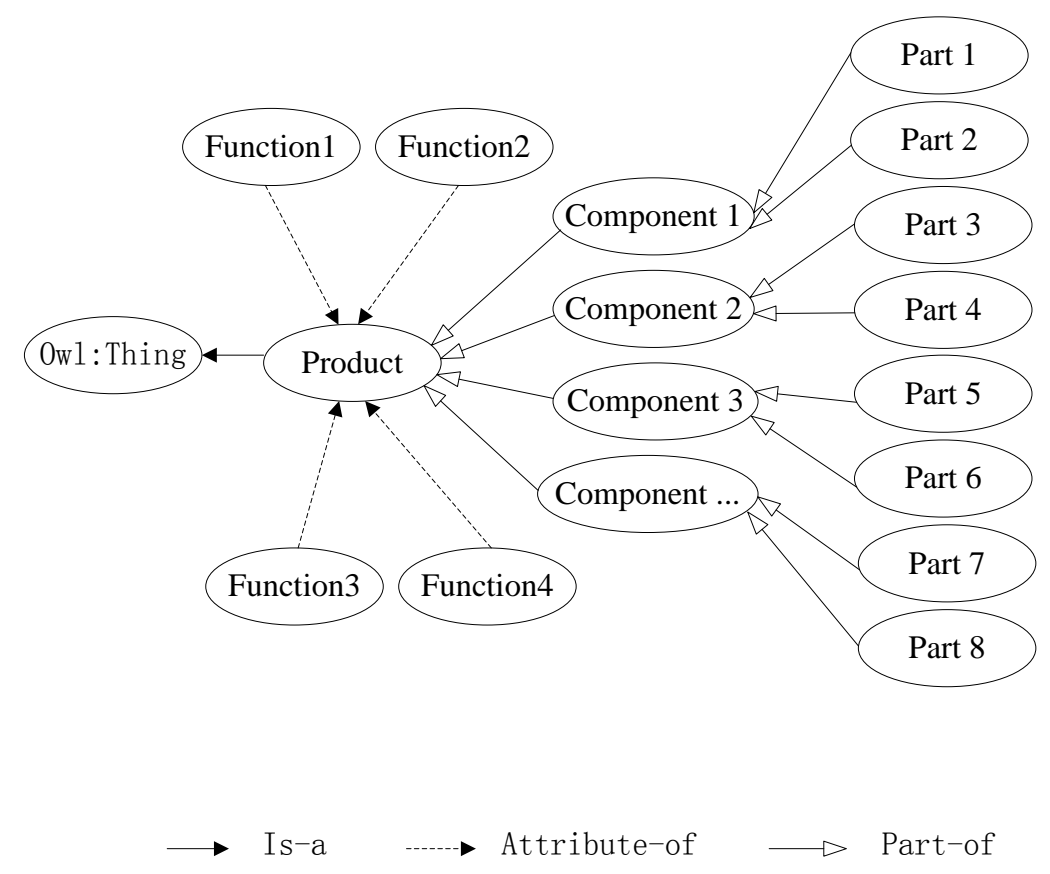

Fig.3 Ontology model of product concept

\subsection{Product conceptual design model based on tacit knowledge}

Based on the analysis of the previous 3 sections, the product conceptual design model based on the user's tacit knowledge is obtained. As shown in figure 4:

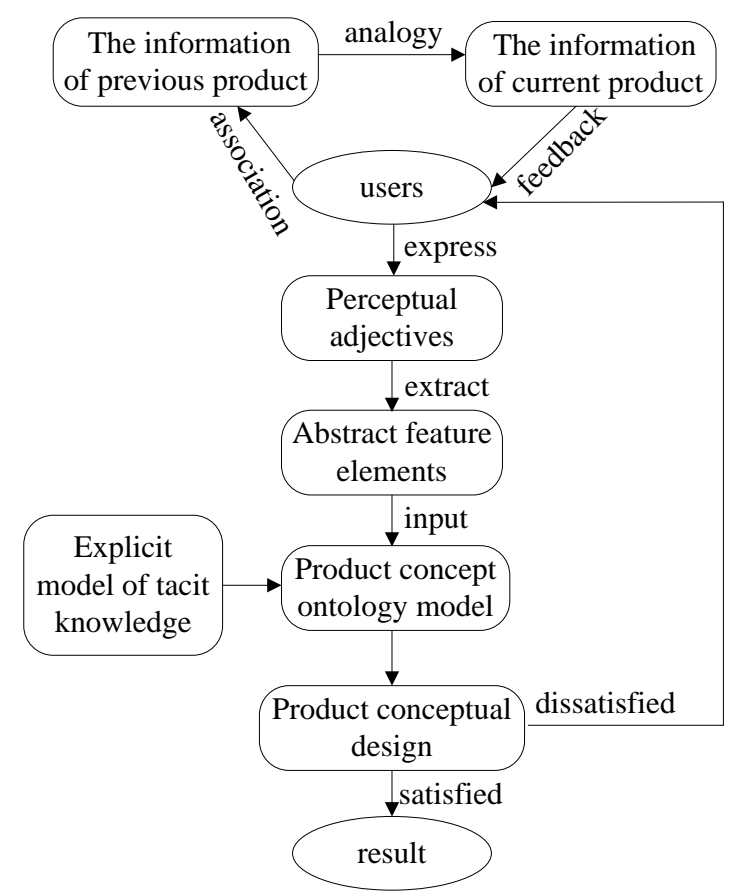

Fig.4 Product conceptual design model based on user's tacit knowledge

Acquisition of tacit knowledge: Before users receive the product information, the first thing to think of the 
previous product information, after receiving the product information, and the previous product information, and the feedback generated by this comparison information to users of the brain; after the user to use all kinds of adjectives to express their sense of the evaluation of the product that is, tacit knowledge.

The explication of tacit knowledge and its application in product conceptual design: After obtaining the tacit knowledge of the user, the designer extracts the abstract feature elements from the product, and inputs them into the input product conceptual ontology model to obtain the explicit knowledge.

Evaluation of product conceptual design: Designers can use product conceptual design of product conceptual design are compared with the former, if the scheme now than before the scheme has certain innovation and practicability, the output results; if compared to the previous scheme without innovation practical, is the new test.

\subsection{Acquisition of tacit knowledge}

\section{Experiment}

In this paper, a small advertisement cleaning machine as an example, the city of small advertising gradually spread, more and more city sanitation workers before the use of an effective and convenient tool to replace knife, shovel and other traditional tools for small ads use clean, so that small ads become cleaning machine more and more, so this paper selects a small advertisement cleaning machine as the research object, more value and significance.

Before the experiment, 20 groups of preset adjectives, through an online questionnaire and by consulting experts, and finally through the comprehensive analysis of designers, selected the following 4 groups of adjectives as user evaluation vocabulary, as shown in table 1 :

Table 1 Adjective pairs

\begin{tabular}{|l|l|}
\hline No. & Adjective pairs \\
\hline 1 & Simple-Complex \\
\hline 2 & Lightweight-Cumbersome \\
\hline 3 & Delicate-Rough \\
\hline 4 & Ordinary-Distinctive \\
\hline
\end{tabular}

In this part, we use the method of questionnaire to get the user's evaluation.

The subjects were randomly selected 20 urban sanitation workers with more than 3 years of experience, including male and female, with a total of 8 women;

Test materials: small advertising machine, a set of 4 pairs of adjectives;

Requirements: to test the subjects introduced the rules, remove small ads cleaning machine nameplate, the subjects were asked to separate, according to their own sense and image, select check in 4 groups of adjectives; Results: according to the data obtained, as shown in Table 2, statistical analysis, table "1" for the user to select group of adjectives in front of a " 0 " on each user to select adjectives behind one, for example, in the simple complex, "1" on behalf of simple the "0 Represents" complex.

Table 2 Test data (part)

\begin{tabular}{|l|l|l|l|l|}
\hline \multirow{2}{*}{ Users } & \multicolumn{4}{|c|}{ The number of adjective pairs } \\
\cline { 2 - 5 } & 1 & 2 & 3 & 4 \\
\hline U1 & 1 & 1 & 1 & 1 \\
\hline U2 & 1 & 1 & 0 & 1 \\
\hline U3 & 1 & 1 & 1 & 0 \\
\hline U4 & 1 & 1 & 0 & 1 \\
\hline U5 & 1 & 1 & 1 & 1 \\
\hline
\end{tabular}

Finally, the test shows that the choice of "concise" has 18 ; select the "light" has 20; select the "refined" has a total of 15; select the "ordinary" has a total of 16.

Therefore, we finally choose the "lightweight", "concise", "ordinary", "delicate" as the user's tacit knowledge.

\subsection{Explicit user knowledge and product conceptual design}

1. Extract the abstract features of the product from the user's perceptual adjectives, as shown in figure 5: 


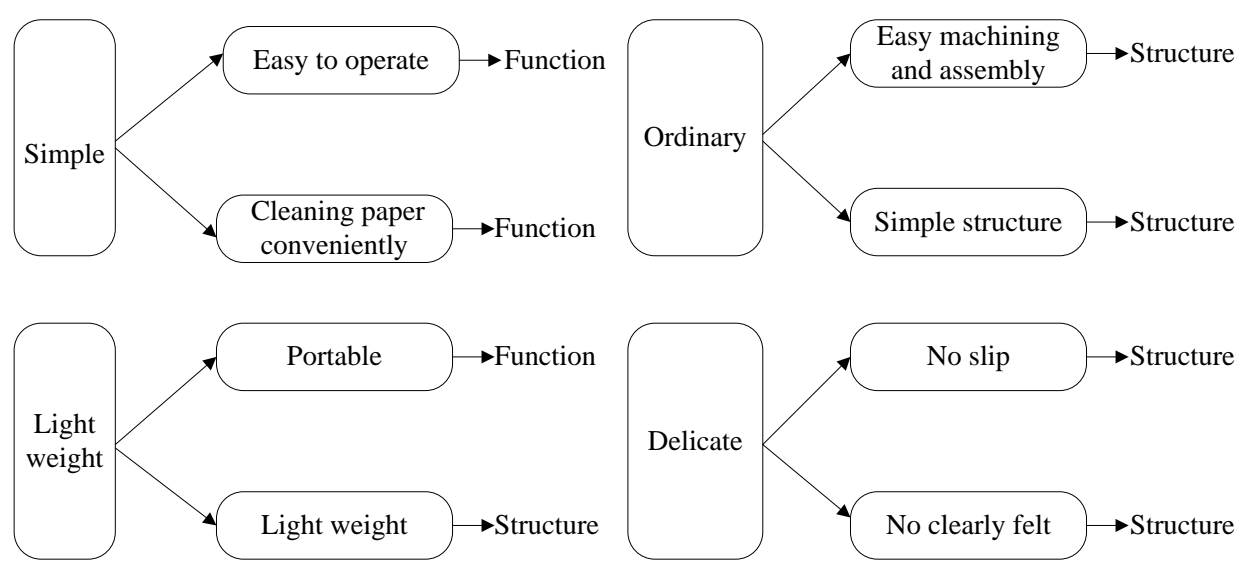

Fig.5 Extraction of the abstract feature elements from the users ' perceptual adjectives

2. By the product concept ontology model, the characteristics of the product will be sorted out, improved and transformed into a small advertising machine to clean the body, so as to achieve the final tacit knowledge of the dominant. As shown in figure 6:

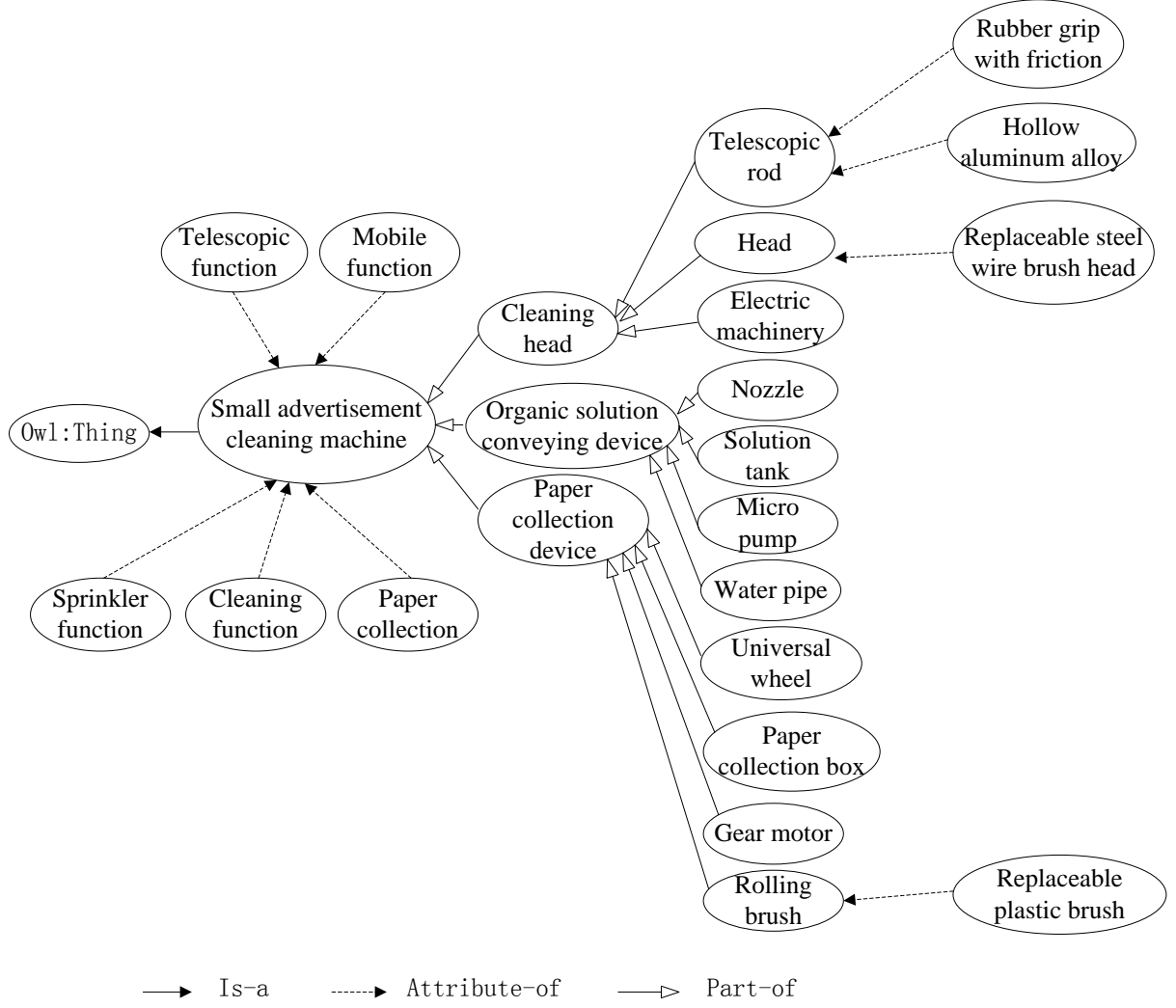

Fig.6 Conceptual model of small advertisement cleaning machine

Technical analysis: principle of telescopic function: is the telescopic rod structure for carrier so as to realize the telescopic function; mobile function: to achieve universal wheel structure for carrier mobile function; function: water spray function to water pipes, sprinkler, micro pump and solution box structure so as to realize the small advertisement carrier cleaning machine; cleaning function: with a brush head, a motor, a telescopic rod structure for carrier so as to realize the small ads cleaning machine cleaning function; paper collection function: a universal wheel, paper collection box, rolling brush is a structure and Realization of small advertising carrier cleaning machine paper collection function motor and reducer.

Multi function cleaning machine assembly small advertising diagram as shown in Figure 7: the cleaning machine is composed of three parts, respectively: cleaning head, conveying device and paper collecting device of organic solution. The cleaning head comprises a telescopic rod and a brush head, a motor, the telescopic rod is a hollow Aluminum Alloy system, can through the pipes, scalable, the top end of the telescopic rod can be connected with the nozzle, the brush head for wire brush head can be replaced easily detachable; organic solution 
conveying device comprises a nozzle, a liquor box, a micro water pump, water pipes, wherein the solution tank liquid can be water, can also be a cleaning solution; paper collecting device comprises a universal wheel, rolling brush, paper collection box, gear motor, gear motor which directly drives the rolling brush to rotate, and by moving the wheel to realize collecting confetti to million. Complete the concept design of small advertisement cleaning machine.

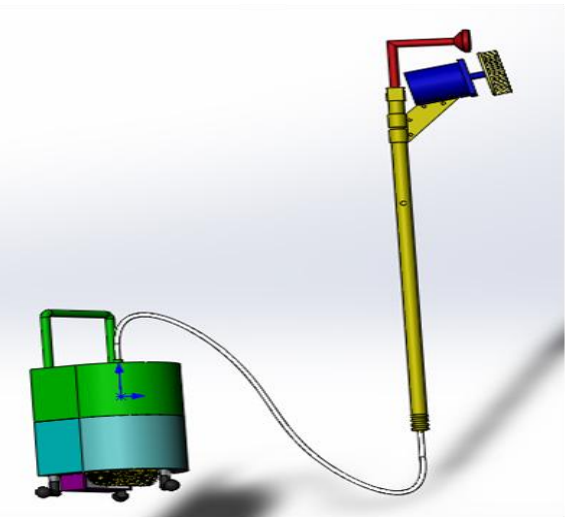

Fig.7 The assembly diagram of small advertising cleaning machine

\section{Conclusion}

In order to improve the quality of product conceptual design, a method for the application of tacit knowledge in product conceptual design is put forward. This article is summarized in the following two aspects:

(1) Users' tacit knowledge is an important source of product conceptual design. The city sanitation workers for the subjects, emotional adjectives, and starting from the cognition and emotion of the user, allowing users to understand their use according to the perceptual given adjectives give the product evaluation, so as to obtain the users tacit knowledge, tacit knowledge and understanding of the product design according to the design division will get to the finish, complete the user implicit conversion of knowledge to product feature abstraction.

(2) The concept ontology model of product is established, and the abstract feature elements of the transformed product are input into the model, so as to realize the dominance of tacit knowledge.

\section{Reference}

[1]. Jacqueline Senker. The Contribution of Tacit Knowledge to Innovation[J]. AI \& Soc, (1993) 7:208-224.

[2]. Jan Andreasik. A Case-Base Reasoning System for Predicting the Economic Situation of Enterprises-Tacit Knowledge Capture Process[J]. Computer Recognition Systems, 2007, 45, 718-730.

[3]. OSGOOD C E, SUCI CJ, TANNENBAUM PH. The measurement of meaning[M]. Urbana : university of Illinois press, 1957.

[4]. ZHAI Lianyin, KHOO L P,ZHONG Zaowei. A rough set based decision support approach to improving consumer affective satisfaction in product design[J].International Journal of Industrial Ergonomics,2009,39(2):295-302.

[5]. Luo Wei, Pan Yunhe, Zhu Shang. Implicit knowledge representation in product design based on graphic thinking [J]. Chinese Journal of mechanical engineering, 2007,43 (6): 93-98.

[6]. Liu Zheng, Lu Na, sun Lingyun. Tacit knowledge acquisition method for conceptual design process [J]. Chinese Journal of mechanical engineering, 2011,47 (14): 184-191.

[7]. Chen Youling, Xiao Yingjiao. Key technology research progress and Countermeasures of [J]. technology for obtaining tacit knowledge in product design, 2011,28 (19): 121-124.

[8]. Luo Shijian, Zhu Shang, Tian Fang, et al. Research status and development of user's tacit knowledge in product design [J]. computer integrated manufacturing system, 2010,16 (4): 673-688.

[9]. Jia Jia. Research on Tacit Knowledge Management Based on case based reasoning [J]. Sweeping the Management over, $2011167-168$.

[10]. Dong Jinxiang. Semantic based service oriented knowledge management and processing [M]. Zhejiang: Zhejiang University press, 2009.

[11]. LIZ, RASKIN V, RAMANI K. Developing engineering ontology for information retrieval [J].Journal of Computer and Information Science in Engineering, 2008, 8 (1) : 1-13. 